\title{
Empirical scaling of sawtooth period for onset of neoclassical tearing modes
}

\author{
IT Chapman ${ }^{1}$, RJ Buttery ${ }^{2}, \mathrm{~S} \mathrm{Coda}^{3}, \mathrm{~S} \mathrm{Gerhardt}^{4}$, JP Graves ${ }^{3}$, DF Howell ${ }^{1}$, A Isayama ${ }^{5}$, RJ La \\ Haye $^{2}$, Y Liu ${ }^{6}$, P Maget ${ }^{7}, \mathrm{M}$ Maraschek ${ }^{8}, \mathrm{~S} \mathrm{Sabbagh}^{9}$, O Sauter ${ }^{3}$, and the ASDEX Upgrade, DIII-D, \\ HL-2A, JT-60U, MAST, NSTX, TCV and Tore Supra Teams and JET-EFDA Contributors* \\ ${ }^{1}$ EURATOM/CCFE Fusion Association, Culham Science Centre, Abingdon, Oxon OX14 3DB, UK \\ ${ }^{2}$ General Atomics, San Diego, CA, USA \\ ${ }^{3}$ CRPP, Association EURATOM/Confédération Suisse, EPFL, 1015 Lausanne, Switzerland \\ ${ }^{4}$ PPPL, Princeton University, PO Box 451, Princeton, NJ 08543, USA \\ ${ }^{5}$ Japan Atomic Energy Agency, Naka, Ibaraki-ken 311-0193 Japan \\ ${ }^{6}$ Southwestern Institute of Physics, PO Box 432, Chengdu, People's Republic of China \\ ${ }^{7}$ CEA, IRFM, F-13108 Saint Paul-lez-Durance, France \\ ${ }^{8}$ MPI fur Plasmaphysik, EURATOM-Ass D-85748 Garching, Germany \\ ${ }^{9}$ Columbia University, New York, USA and \\ * See the Appendix of F. Romanelli et al., Fusion Energy 2008 (Proc. 22nd Int. Conf. Geneva, 2008) IAEA, (2008)
}

\begin{abstract}
Experimental observations from a range of tokamaks show that neoclassical tearing modes (NTMs) are triggered at lower plasma pressure when the sawtooth period is longer. A multi-machine database from nine tokamaks has been established in order to extrapolate the acceptable sawtooth period to avoid triggering NTMs in ITER. It is found that the governing physics is best compared between machines by normalising the sawtooth period to the resistive diffusion time and using the normalised beta as a measure of performance and global stability. A multi-parameter power scaling is determined from regression analysis of the complete dataset and compared favourably with experimental data from a number of machines.
\end{abstract}

PACS numbers: $52.55 \mathrm{Fa}, 52.35 \mathrm{Py}$

The neoclassical tearing mode (NTM) is one of the most critical limiting plasma instabilities for baseline scenarios in ITER and for next-step devices. The NTM is driven by a helical perturbation that reduces the local bootstrap current - a self-generated plasma current in regions of strong pressure gradients and low collisionality. The local reduction in bootstrap current results from the pressure profile flattening in the presence of a magnetic island. NTMs enhance cross-field transport and degrade confinement, sometimes even leading to plasma terminations. The saturated islands often represent the operational limit to achieving low collisionality, high pressure, high confinement and long pulse discharges. Consequently, there is much effort to develop effective methods to avoid or ameliorate NTMs. Whilst direct NTM suppression can be facilitated by driving local current in the island in order to replace the bootstrap current, indirect avoidance methods are also possible.

The NTM is a metastable mode which requires a 'seed' perturbation in order to be driven unstable and grow [1], except at very high plasma pressure where the linear tearing stability index $\Delta^{\prime}$ can become large and positive as it approaches a pole discontinuity [2]. Various effects have been proposed to prevent NTM growth for small island widths, namely (i) incomplete pressure flattening which occurs when the connection length is long compared to the island width [3], (ii) ion polarisation currents arising due to finite orbit width $E \times B$ drifts occurring for ions and electrons across the island region $[4,5]$, which act to replace the missing bootstrap current, and (iii) curvature effects $[6,7]$. Consequently, NTM growth is generally prohibited in the absence of a sufficiently large seed island in the plasma. Whilst this seed may be caused by edge localised modes (ELMs) [8, 9] or fast particle-driven fishbones [10], the trigger of most concern is the sawtooth oscillation which typically triggers the NTMs at lower plasma pressures [10]. It has been shown on JET that sawteeth are more likely to trigger NTMs when the sawtooth period is long [11-13]. Many theories have been proposed to explain how the sawtooth crash triggers the NTM, including magnetic coupling [14], nonlinear 'three-wave' coupling [15], changes in the classical tearing stability due to current redistribution inside $q=1$ [16-18] or changes in the rotation profile resulting in a reversal of the ion polarisation current [19] in the modified Rutherford equation governing NTM stability [20]. These models predict that the salient features of the sawtooth crash that should determine the onset of the NTM are the amplitude of the magnetic perturbation, the coupling to the NTM rational surface and any shielding effects such as rotational screening or diamagnetic effects. However, empirical observation and neural network analysis have determined that the sawtooth period shows far stronger correlation to the triggering of the NTM than the sawtooth amplitude [11, 13, 19, 21].

Although the coupling physics which underlies the seeding of the NTM by the sawtooth crash remains poorly understood, the empirical observation that deliberately increasing the sawtooth frequency helps to avoid triggering NTMs is now universally accepted and routinely used as a method for NTM mitigation. This can be achieved by increasing the magnetic shear at the $q=1$ surface or by tailoring the distribution of a fast ion population around $q=1$ 
to reduce the potential energy of the internal kink mode (see [22] and references therein). However, predictions for controlling sawteeth in ITER in order to avoid triggering NTMs are predicated upon knowing an acceptable sawtooth period that is unlikely to seed NTMs. An analytical assessment of the sawtooth period in ITER was proposed in reference [23] where heuristic linear stability thresholds for the sawtooth crash were simulated using a 1-d transport solver including a full reconnection model, predicting a sawtooth period of 100 s with a $q=1$ radius of $50 \%$ of the plasma minor radius. Indeed, a similar answer is arrived at by extrapolating monster sawteeth observed on JET [24] by the resistive diffusion time to ITER [25]. Since then, the same crash trigger model has been implemented in more accurate transport codes. Modelling using TSC [26] with H-mode profiles given by either the multi-mode model [27] or the Gyro-Landau fluid model GLF23 [28] predicted 50s sawtooth period for the complete reconnection model and 2-3 times shorter periods for partial reconnection, with a $q=1$ radius of $42 \%$ of the minor radius (which agrees well with the inversion radius predicted in BALDUR modelling [29]). Finally, time-dependent integrated predictive modelling with the PTRANsP code predicted a sawtooth period much less than 50s [30]. All of these predictions entail sawteeth with a quiescent period much longer than the energy confinement time. Whilst the sawteeth have a negligible effect on the stored energy or the rate of neutron production, it is the possibility that sawteeth with $\tau_{s t} \gg \tau_{E}$ could trigger NTMs which is of primary concern. The issue of whether a sawtooth period in the range of 20-50s will avoid triggering NTMs is currently poorly understood, and so a multi-machine empirical scaling is presented in this paper in order to provide some basis for extrapolation and design of sawtooth control actuators in ITER.

A database of plasma parameters has been established for discharges which exhibit sawteeth, including both crashes which trigger NTMs and those which do not. This dataset contains details for over 200 shots from nine tokamaks; namely ASDEX Upgrade, DIII-D, HL-2A, JET, JT-60U, MAST, NSTX, TCV and Tore Supra. In order to be able to differentiate between the different dominant physics mechanisms in each case, it is necessary to retain a significant number of plasma parameters, including, but not limited to: the sawtooth period $\tau_{s t}[\mathrm{~ms}]$, the NTM poloidal and toroidal mode numbers $(m, n)$, the major radius $R_{0}[\mathrm{~m}]$, the minor radius $a[\mathrm{~m}]$, the elongation $\kappa$, the triangularity $\delta=\left(\delta_{\text {up }}+\delta_{\text {low }}\right) / 2$, the magnetic field $B_{v a c}[\mathrm{~T}]$, the plasma current $I_{p}[\mathrm{MA}]$, the radial position of the q=1 surface $r_{1} / a$ found from the inversion radius or magnetic equilibrium reconstruction, the normalised beta $\beta_{N}=2 \mu_{0}\langle p\rangle a / B_{v a c} I_{p}$ where $\langle\cdots\rangle$ is a volume averaged quantity, the poloidal beta $\beta_{p}=2 \mu_{0}\langle p\rangle / B_{a}^{2}$ where $B_{a}=\mu_{0} I_{p} / \sqrt{2 V / R_{0}}$ and $V$ is the plasma volume, the electron density at the magnetic axis $n_{e 0}\left[\mathrm{~m}^{-3}\right]$, the internal inductance $l_{i}=\left\langle B_{p}^{2}\right\rangle \sqrt{2 V / R_{0}} /\left(\mu_{0} I_{p}\right)$, the heating powers $P_{N B I}[\mathrm{MW}], P_{I C R H}[\mathrm{MW}]$ and $P_{E C R H}[\mathrm{MW}]$, the line averaged density $\bar{n}_{e}\left[\mathrm{~m}^{-3}\right]$, the line-averaged effective charge $Z_{\text {eff }}$, the electron temperature on axis $T_{e 0}[\mathrm{eV}]$ and the toroidal rotation speed on axis $v_{\phi 0}[\mathrm{~km} / \mathrm{s}]$. From these parameters, various derived quantities are also calculated. The Alfvén speed is defined as $v_{A}=B_{v a c} / \sqrt{\mu_{0} n_{i} m_{i}}$ where $m_{i}$ is the ion mass. The Alfvén time is $\tau_{A}=R_{0} / v_{A}$. The poloidal ion Larmor radius is $\rho_{\theta i}=v_{t h i} m_{i} / e B_{\theta}$ where $B_{\theta}=\mu_{0} I_{p} / 2 \pi a$ and $v_{t h i}=\left(k T_{i} / m_{i}\right)^{1 / 2}$. The power required to access H-mode confinement [31] is given by

$$
P_{L H}[M W]=0.0488 \mathrm{e}^{ \pm 0.057} n_{20}^{0.717 \pm 0.035} B_{v a c}^{0.803 \pm 0.032} S^{0.941 \pm 0.019}
$$

where $n_{20}=n_{e} / 10^{20}$ and $S$ is the plasma surface area. The electron collisionality is given by [32]

$$
\nu_{e *}=6.921 \cdot 10^{-18} \frac{R_{0} n_{e 0} Z_{e f f} \ln \Lambda_{e}}{T_{e 0} \epsilon_{1}^{3 / 2}}
$$

where the electron density $\left[\mathrm{m}^{-3}\right]$ and temperature $[\mathrm{eV}]$ are taken at the magnetic axis rather than the $q=1$ surface for simplicity, $\epsilon_{1}=r_{1} / R_{0}$ and $\ln \Lambda_{e}=31.3-\ln \left(\sqrt{n_{e 0}} / T_{e 0}\right)$. The Spitzer resistivity is defined as

$$
\eta_{\text {spitz }}=Z_{\text {eff }}\left(0.58+\frac{0.74}{0.76+Z_{\text {eff }}}\right) \frac{17}{1.9012 \cdot 10^{4} T_{e}^{3 / 2}}
$$

The geometrical effects on conductivity in the collisionless limit are incorporated using the effective trapped fraction, defined as $[32]$

$$
f_{t e f f}\left(\nu_{e *}\right)=\frac{f_{t}}{1+\left(0.55-0.1 f_{t}\right) \sqrt{\nu_{e *}}+0.45\left(1-f_{t}\right) \nu_{e *} / Z_{e f f}^{3 / 2}}
$$

and the trapped fraction is given by

$$
f_{t}=1-\frac{\left(1-\epsilon_{1}\right)^{2}}{\sqrt{1-\epsilon_{1}^{2}}\left(1+1.46 \sqrt{\epsilon_{1}}\right)}
$$



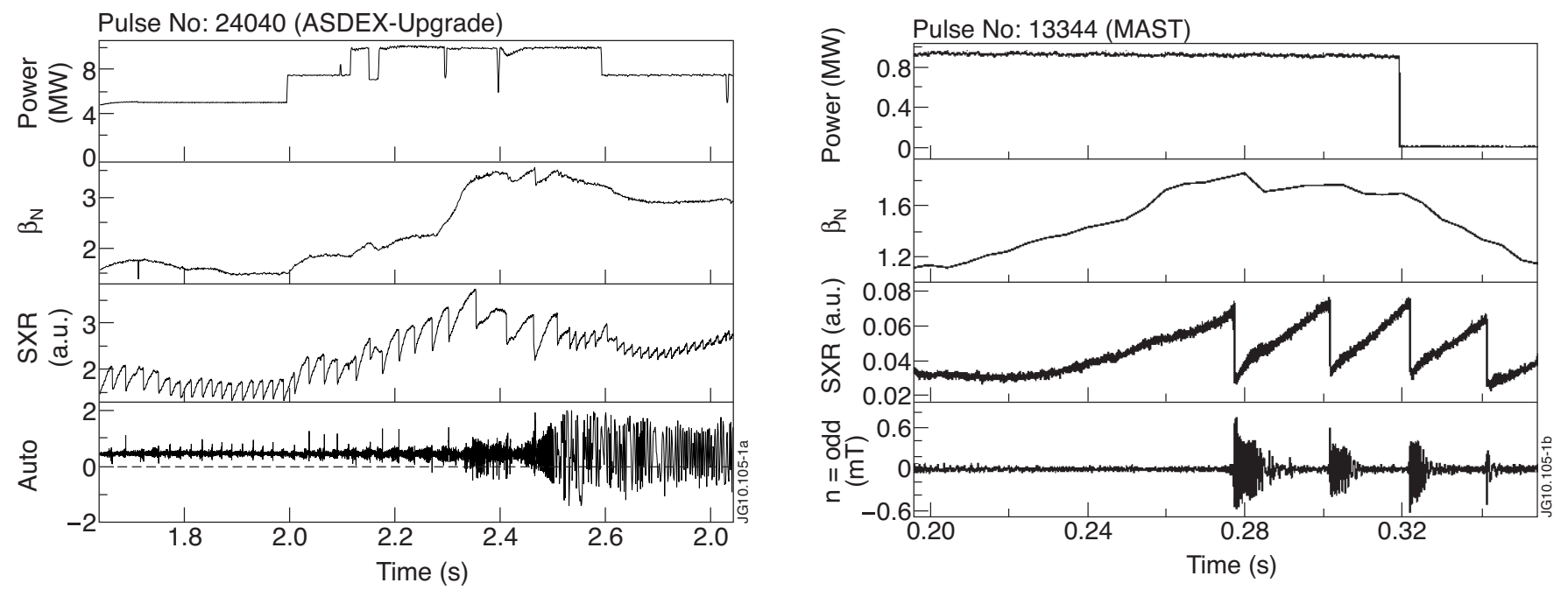

FIG. 1: The time traces of the NBI power, $\beta_{N}$, Soft X-ray emission and magnetics amplitude for (left) a $m / n=3 / 2$ NTM triggered by a sawtooth crash discharge 24040 in ASDEX Upgrade and (right) a series of 2/1 NTMs triggered in discharge 13344 in MAST

Using equations 3 and 4, the neoclassical resistivity can be defined as

$$
\eta_{\text {neo }}=\eta_{\text {spitz }}\left[1+f_{\text {teff }}\left(-\left(1+\frac{0.36}{Z_{\text {eff }}}\right)+\frac{0.59}{Z_{\text {eff }}} f_{\text {teff }}-\frac{0.23}{Z_{\text {eff }}} f_{\text {teff } f}^{2}\right)\right]
$$

Finally, the resistive diffusion time can be derived from equation 6 as

$$
\tau_{r}=\frac{\mu_{0} r_{1}^{2}}{1.22 \eta_{\text {neo }}}
$$

For all of these derived quantities the assumption that $T_{i}=T_{e}$ and $n_{i}=n_{e}$ is made since electron pressure data is available for all discharges.

Naturally, comparing discharges between a large range of tokamaks means that the database contains a wide range of plasma shapes, $q$-profiles, fast ion pressures and fast ion distribution functions, all of which will influence the sawtooth behaviour. Similarly, the different $q$-profiles, and thus different magnetic shear between rational surfaces, as well as the different rotation profiles will undoubtedly influence the coupling between the sawtooth oscillations at $q=1$ and the NTM at higher rational surfaces. The database also incorporates triggered NTMs at three different rational surfaces, namely $q=4 / 3,3 / 2,2 / 1$. However, retaining such a wide range of plasma parameters means that a "safe" operating space, where sawteeth are less likely to trigger NTMs, can be inferred.

Figure 1 shows typical examples of a $m / n=3 / 2$ NTM being triggered by a sawtooth crash in ASDEX Upgrade and a 2/1 mode being triggered in MAST. The ASDEX Upgrade example typifies the problem of triggering NTMs: In order to increase the $\beta_{N}$ for increased fusion performance, progressively more heating power is applied. However, as well as increasing the plasma pressure, this introduces a population of energetic particles which strongly stabilises the sawtooth. As such, the sawtooth period increases, and at sufficiently high $\beta_{N}$ and $\tau_{s t}$, an NTM is triggered, which results in a significant collapse in the performance. Similarly in MAST, the first two sawtooth-triggered NTMs result in a marked decrease in the plasma pressure despite constant heating power.

This inability to operate at high plasma pressure when the sawtooth period is long without the aid of active control is borne out by the complete database, as illustrated in figure 2 . There are no points in the (high $\beta_{N}$, high $\tau_{s t} / \tau_{r}$ ) operating space. One possible explanation is that as the sawtooth period increases, NTMs are more readily triggered, deteriorating confinement and precluding high plasma pressures. Conversely, when the sawtooth period is short, various mechanisms can be responsible for the ultimate triggering of the NTMs, as the coupling is more sensitive to the local rotation shear and $q$-profile nuances for a smaller seed perturbation, resulting in an increased scatter in the data and a wide range of plasma pressures at which NTMs are triggered. It should be noted that whilst there are high-power NBI-only heated discharges in JET and ASDEX Upgrade with large $\tau_{s t} / \tau_{r}$ which have $H_{98(y, 2)}$-factor significantly less than one (since the sawtooth crash has occurred within an energy confinement time of an L-H transition) implying that the plasma could have accessed higher $\beta_{N}$ if $H_{98(y, 2)}$ increased as expected in the absence of 


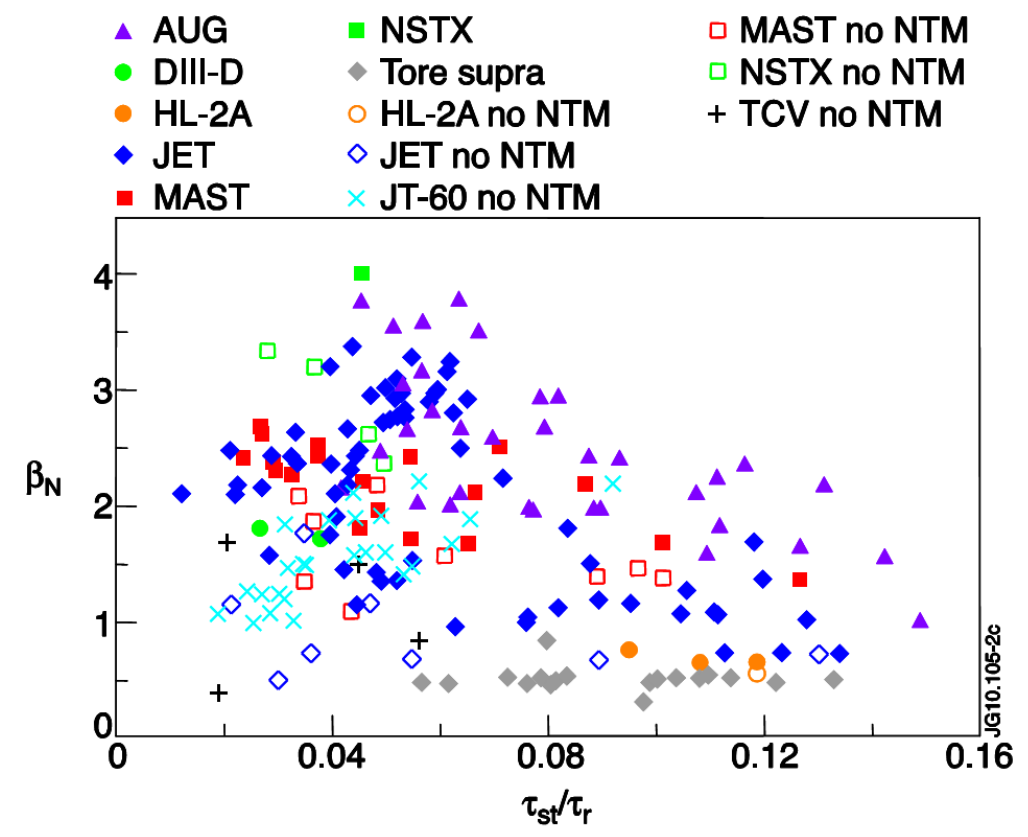

FIG. 2: $\beta_{N}$ at the NTM onset with respect to the sawtooth period normalised to the resistive diffusion time (here all $(m, n)$ NTMs are included).

the sawtooth-triggered NTM, there are also cases in the database at long $\tau_{s t}$ which have $H_{98(y, 2)}$-factor greater than or equal to one, indicating that they are $\beta_{N}$ limited by low input power. Whilst the exact $\beta_{N}$ at which the NTM is triggerable depends on the trajectory of the discharge in the $\left(\beta_{N}, \tau_{s t} / \tau_{r}\right)$ operating space, the points which mark the boundary between avoiding and triggering NTMs are the most important since these delineate the acceptable regime of operation.

In figure 2 the sawtooth period has been normalised to the resistive diffusion time as calculated using equation 7. The dynamics which determine when the sawtooth crash will occur (in the absence of any deliberate sawtooth control actuators) are predominantly determined by the evolution of the $q$-profile, particularly of the radial position of the $q=1$ surface and the local magnetic shear at $q=1$ [23]. Since these quantities evolve on the timescale of the resistive diffusion in the plasma core, the sawtooth period has been normalised accordingly. The excellent machine data collapse achieved with this timebase adds credence to this normalisation, especially as such a comparison cannot be made when the energy or momentum confinement time (governing stabilisation or coupling effects from rotation or fast ions, for instance) or the Alfvén time (the growth time of the ideal internal kink mode) is used. The quantification of the plasma performance is somewhat more subtle. From an NTM stability view point, one would think that the poloidal beta should dominate whether the sawtooth crash is followed by NTM growth or not [3, 4, 20]. However, in the majority of the discharges in the database, the triggered NTM is largely metastable, which is to say that

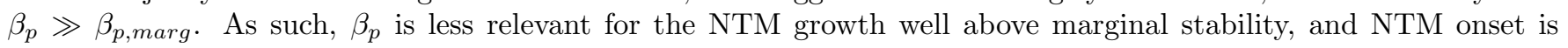
dominantly determined by the occurrence of a sufficiently large seed island. However, what constitutes a sufficiently large island size is a complicated matter, but it can be associated with global MHD stability of the plasma, which can be parameterised by $\beta_{N}$. The stability of ideal long wavelength modes (such as the internal kink that drives the sawtooth crash) depends on pressure gradients volume averaged within the rational surface at which the mode's eigenfunction is maximised (ie a local evaluation of $\beta_{p}$ ). This is naturally independent of the global profiles, such as an edge pressure pedestal, whereas $\beta_{N}$ is elevated in H-mode. Whilst $\beta_{N}$ is often considered as a proxy for stability thresholds for small scale MHD instabilities (such as interchange or ballooning modes), it also governs global stability, such as the external kink mode or infernal mode limits. Of course, it is the coupling of the $1 / 1$ mode to a higher $m / n$ mode which must dominate whether a sawtooth crash seeds an NTM, but it seems that using $\beta_{N}$ as a measure of "global" stability can distinguish regions of operating space whereby an NTM is triggered or otherwise. A number of 


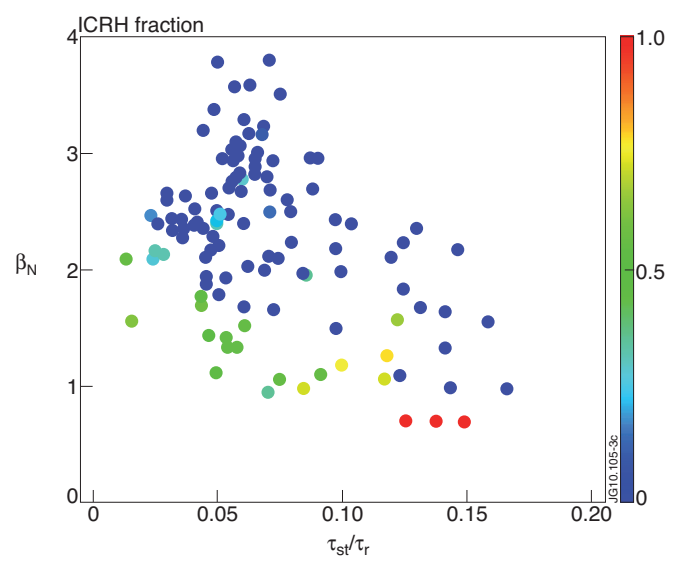

FIG. 3: $\beta_{N}$ at the NTM onset with respect to the sawtooth period normalised to the resistive diffusion time for the JET database. The colours indicate the fraction of auxilliary heating power provided by resonant frequency heating.

other, in many cases more intuitive, parameters have been considered, but none considered here exhibit any trends, nor achieve a parameter space occupied by all machines as observed in figure 2. Analysing the data in terms of both $\beta_{p}$ and $\rho_{\theta}$ leads to a machine separation in the data, and notably no distinction between sawteeth which triggered NTMs and those which did not. It should be noted here that $\beta_{p}$ has been evaluated globally since a local $\beta_{p}$ at $q=1$ cannot be evaluated with certainty because of the errors on dependent quantities such as the total pressure gradients in the core and $r_{1}$ values. Considering the critical island width for NTM growth according to the model in reference [3] or the critical seed island width in reference [33] did not exhibit any distinction concerning whether an NTM is triggered or not either. Finally, normalising $\beta_{N}$ by the auxiliary heating power or by a number of other profile dependent quantities, such as $l_{i}$, pressure peaking or density peaking did not lead to any improvement in the trends.

These normalisations cannot remove specific traits of each machine which will naturally bias the data. For instance, the HL-2A and Tore Supra plasmas have a circular cross-section which will naturally lead to different internal kink stability and coupling physics compared to the shaped plasmas of the other devices. JT-60U typically operates at high $\beta$ with early heating to delay current penetration and avoid sawteeth by keeping $q>1$. This means that all sawtooth data is from plasmas operated at low pressures. Similarly NSTX has few instances of sawtooth-triggered NTMs as the safety factor is usually kept above unity to access high performance plasmas. The DIII-D data is in ITER-like discharges with $\beta$ feedback and cryopumps to control density, whereas the MAST discharges have sawteeth when the density is high, producing lower temperature and faster current diffusion resulting in $q<1$.

Previous JET data [11] suggested that NTMs triggered by long sawtooth periods were only observed in plasmas with a significant fraction of heating from ion cyclotron resonance heating. However, by normalising the sawtooth period to the resistive diffusion time, this separation is removed, as illustrated in figure 3. Whilst long sawtooth periods can be obtained with less heating power when RF heating is applied, it is important to note that long sawtooth periods which trigger NTMs at low $\beta$ are achieved with NBI heating alone. Indeed, the longest $\tau_{s t} / \tau_{r}$ in the JET dataset is achieved in an NBI-only discharge, facilitating direct comparison with other machines that do not have RF heating. Nevertheless, there is a clear increase in the achievable $\beta_{N}$ as the ICRH fraction reduces across the range of sawtooth periods. This is likely to be due to both the effect on mode stability in the presence of very energetic ions, and also a reduction in the plasma rotation in the absence of significant momentum imparted by neutral beams, which reduces the screening of magnetic coupling between $q=1$ and $q=3 / 2$ surfaces. It should be pointed out that all ICRH-heated discharges in the database utilise co-propagating on-axis RF waves which stabilise the internal kink mode [34, 35]. Plasmas with direct sawtooth control are excluded in order to consider the natural sawtooth period at which an NTM could be triggered. It is also worth noting that in many instances, the longest sawtooth periods are achieved at the first sawtooth crash following the L-H transition, not only because of the improved confinement of energetic particles, but also because of transient effects and changes in the evolution of the plasma profiles.

Notwithstanding the individual constraints of each tokamak, there is still such a significant scatter in figure 2 that it is difficult to draw any conclusion about the permissible sawtooth period in ITER that avoids triggering 


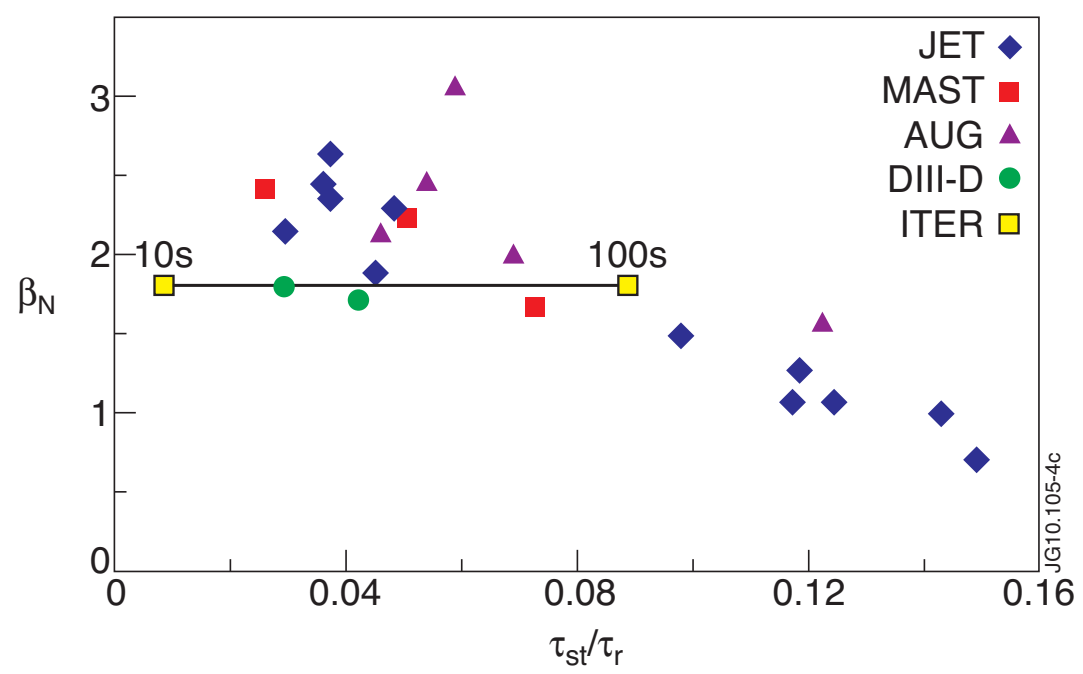

FIG. 4: $\beta_{N}$ at the NTM onset with respect to the sawtooth period normalised to the resistive diffusion time for ITER-like shape, $q=1$ radius and injected power normalised to the $P_{L H}$ threshold. For comparison, ITER Scenario 2 is indicated with sawtooth period ranging from 10 s to $100 \mathrm{~s}$

NTMs. However, as previously explained, the range of machine parameters in the complete database is rather large, encompassing a wide variation in shape, $q$-profiles and heating schemes. Consequently, in order to make a more reliable extrapolation to ITER, a subset of the data has been considered which retains only discharges with ITER like shape $(\delta \in[0.3,0.4]$ and $\kappa \in[1.65,1.85])$, a broad flat $q$-profile with a wide $q=1$ surface $\left(r_{1} / a \in[0.33,0.45]\right)$ and with auxilliary heating power only slightly above the $\mathrm{L}-\mathrm{H}$ threshold given by equation $1\left(P_{\text {aux }} / P_{L H} \in[1.3,1.7]\right)$ as expected in the ELMy H-mode baseline scenario in ITER (Scenario 2) [36, 37]. This reduced database of "ITER-like" sawtoothing discharges is illustrated in figure 4 . It is clear that this subset retains the general trend revealed by the full database, namely that NTMs are triggered at lower $\beta_{N}$ for longer sawtooth periods with respect to the resistive diffusion time.

Also shown in figure 4 is the range of sawtooth periods that could be expected in ITER. A period of 20-50s predicted by transport modelling $[29,30]$ would lie in the range $\tau_{s t} / \tau_{r} \in[0.0178,0.0446]$ which approaches the period at which this empirical extrapolation suggests NTMs would be triggered by the sawtooth crashes at the target plasma pressure of $\beta_{N}=1.8$ in ITER scenario 2. However, if the natural sawtooth period is approximately the same as the critical period for triggering NTMs, there is the opportunity to apply control actuators to sufficiently reduce $\tau_{s t}$ and avoid NTMs, which would not be the case if the natural period was significantly longer than the critical period. Numerical modelling of electron cyclotron driven current predicted in ITER suggests that the magnetic shear at the $q=1$ surface can be increased sufficiently to incur a reduction in the sawtooth period of approximately $30 \%$ [38, 39], although it should be noted that these transport simulations are highly sensitive to the choice of the numerical coefficient used in the linear crash criterion from [23].

Finally, it is possible to produce a simplistic power-law scaling derived from the least-squares fit of the $\beta_{N}$ for NTM onset with respect to each parameter in the complete database. For this regression analysis all the cases of sawtooth-triggered NTMs from seven machines (namely ASDEX Upgrade, DIII-D, HL-2A, JET, MAST, NSTX and Tore Supra) are retained. Whilst individual machines do exhibit trends with certain parameters (for instance, Tore Supra shows a strong correlation with amplitude of the perturbation at the $q=1$ radius [40]), only variables exhibiting a strong trend with a good statistical correlation across all machines are retained. This produces a scaling for the critical $\beta_{N}$ at which a sawtooth crash will trigger an NTM, given by

$$
\beta_{N}^{\mathrm{NTM} \text { onset }}=2.614\left(\frac{\tau_{s t}}{\tau_{r}}\right)^{-0.4084} \rho_{\theta}^{0.5721}\left(\frac{P_{a u x}}{P_{L H}}\right)^{0.4204} \bar{n}_{e}\left[10^{19} m^{-3}\right]^{0.4948}
$$

where $\rho_{\theta}$ is the normalised poloidal ion Larmor radius, $\rho_{\theta}=\rho \theta i / r_{1}$. The fit of the predicted $\beta_{N}$ for NTM onset given 


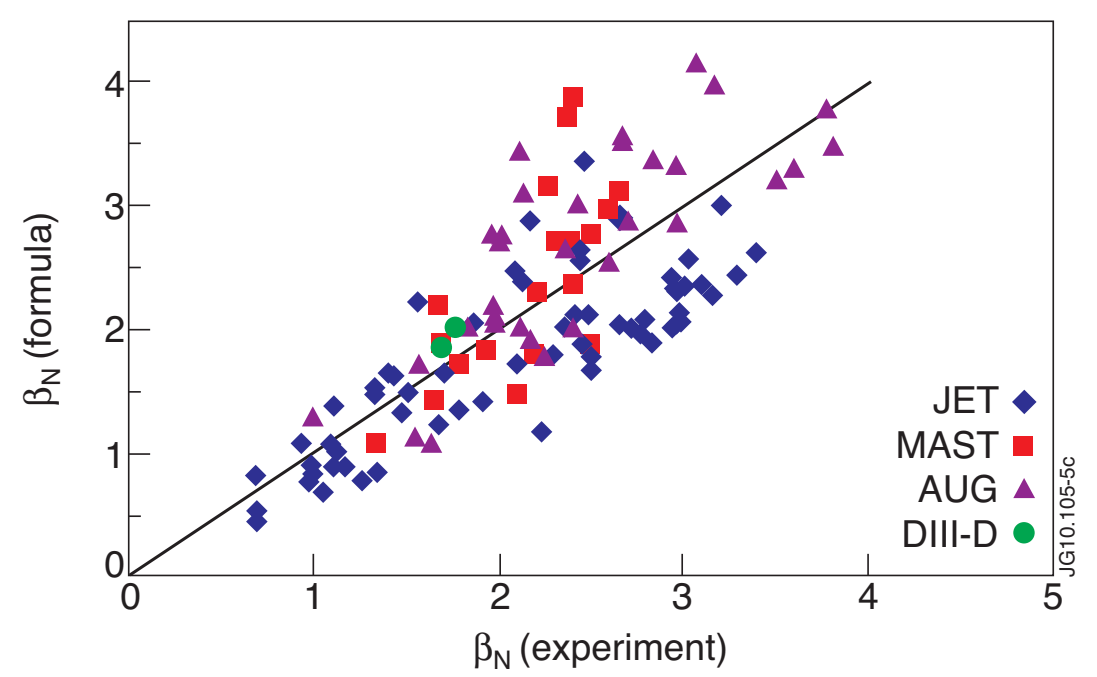

FIG. 5: The $\beta_{N}$ at which an NTM is triggered experimentally compared to the critical $\beta_{N}$ predicted by equation 8 .

by equation 8 compared to the experimental values is shown in figure 5 . The coefficient of determination - which is an indicator of how well the equation resulting from the regression analysis explains the relationship between the variables - is 0.601 (where 1 is perfect correlation). In regression analysis, the coefficient of determination provides a measure of how well future outcomes can be predicted by a model [41]. It should be noted, however, that the variables in the expression for $\beta_{N}^{\text {crit }}$ given by equation 8 are unlikely to be truly independent, since the sawtooth period is determined by complex physical mechanisms which are not fully understood. Finally, we reiterate that in the majority of the discharges in this database, the triggered NTM is largely metastable and NTM onset is dominantly determined by the occurrence of a sufficiently large seed island. Equation 8 suggests that the critical $\beta_{N}$ for triggering an NTM by a sawtooth crash in ITER is 2.09 for a sawtooth period of 50s. At the target operating pressure for ITER Scenario 2 $-\beta_{N}=1.8$ - this scaling law suggests that a sawtooth period of around 70 s will be permissible.

It should be noted that the power-law scaling exponents are determined from a least squares fit to the entire data set of sawtooth-triggered NTMs rather than the marginal points (ie the lower $\beta_{N}$ boundary of the database). Consequently, the scaling is likely to over-predict the achievable $\beta_{N}$. That said, figure 6 shows that the ITER-like dataset compares favourably to the critical $\beta_{N}$ at which a sawtooth crash would be expected to trigger an NTM according to the scaling law in equation 8. Furthermore, when the scaling law is applied to JT-60U and TCV data from figure 2 , the majority of cases are predicted to trigger NTMs only at higher $\beta_{N}$ than that achieved in the experiment, where NTMs were absent. This agreement adds credence to the applicability of this scaling law. Also shown in figure 6 for comparison is the critical $\beta_{N}$ which could be anticipated in ITER baseline scenario for a range of sawtooth periods from 10s to 100s. It is evident that a sawtooth period in the range of 20-50s predicted by transport simulations is predicted to avoid triggering NTMs at the scenario target operating pressure. It is also clear that the critical $\beta_{N}$ for NTM onset increases as the sawtooth period is reduced, highlighting the need for provision of sawtooth control actuators. This scaling law is, of course, only an empirical fitting and not based on any physics model, so its application to future devices should only be for guidance, and certainly not quantitative.

Experimental observation from a number of tokamaks has exhibited triggering of NTMs at lower normalised beta when the sawtooth period is increased. This has serious implications for ITER where the fusion-born $\alpha$ particles are likely to lead to naturally long sawtooth periods [23, 42]. The empirical scaling established through a database including discharges from nine machines suggests that the the intrinsic sawtooth period predicted by transport modelling for baseline scenario operation of ITER is likely to approach the critical period for triggering NTMs. This not only enforces the acute need for actuators for sawtooth control capable of reducing the period sufficiently to avoid the triggering of secondary instabilities, but also suggests that the requirements upon these actuators for destabilisation of sawteeth are likely to be within the bounds of capability of the heating and current drive systems, which would not be the case if the natural period far exceeded the threshold for NTM seeding. 


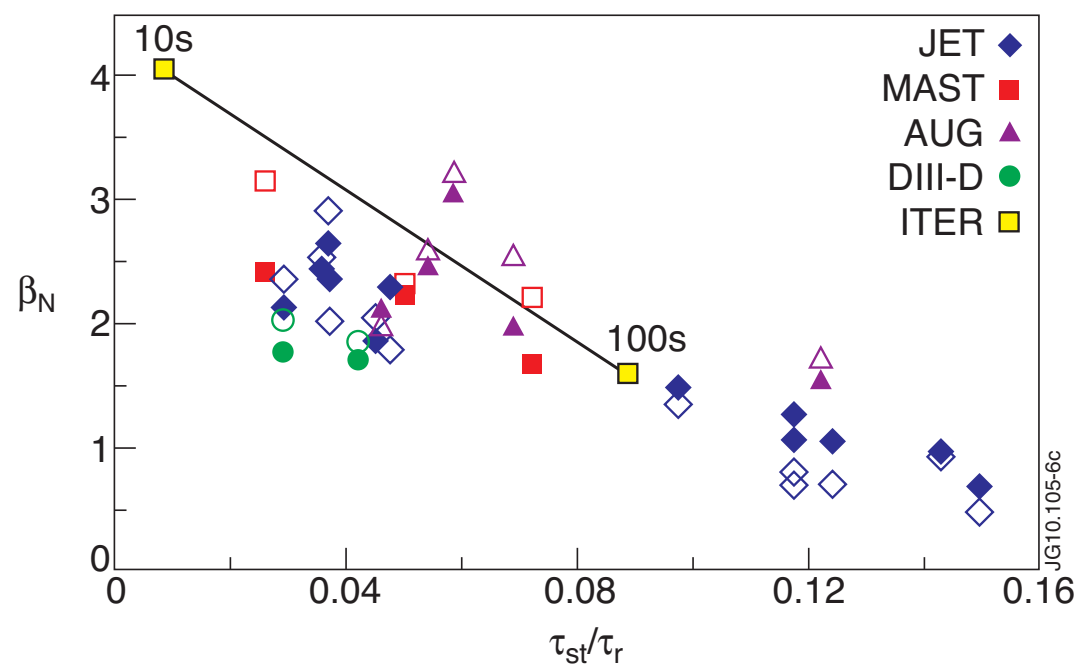

FIG. 6: The $\beta_{N}$ at which an NTM is triggered with respect to the sawtooth period normalised to the resistive diffusion time (filled symbols) for ITER-like shape, $q=1$ radius and injected power normalised to the $P_{L H}$ threshold, compared to the critical $\beta_{N}$ predicted by equation 8 (open symbols). For comparison, ITER scenario 2 as predicted by equation 8 is indicated with sawtooth period ranging from 10 s to 100 s.

IC gratefully acknowledges useful discussions with Drs JW Connor, RJ Hastie and TC Hender. This work was conducted under the auspices of the ITPA MHD Stability Topical Group. This work was partly funded by the United Kingdom Engineering and Physical Sciences Research Council under grant EP/G003955, the European Communities under the contract of Association between EURATOM and CCFE and supported in part by US DOE under contracts DE-AC02-09CH11466, DE-FC02-04ER54698, and DE-FG02-99ER54524. The views and opinions expressed herein do not necessarily reflect those of the European Commission.

[1] R Carrera, RD Hazeltine and M Kotschenreuther 1986 Phys Fluids 29899

[2] DP Brennan et al 2003 Phys. Plasmas 101643

[3] R Fitzpatrick et al, 1995 Phys. Plasmas 2825

[4] HR Wilson et al, 1996 Phys. Plasmas 3248

[5] AI Smolyakov, A Hirose, E Lazzaro, GB Re and JD Callen 1995 Phys Plasmas 21581

[6] M Kotschenreuther, RD Hazeltine and PJ Morrison 1985 Phys Fluids 28294

[7] H Lütjens, JF Luciani and X Garbet 2001 Phys Plasmas 84267

[8] RJ Buttery et al 2008 Phys. Plasmas 15056115

[9] S Gerhardt et al 2009 Nucl. Fusion 49032003

[10] A Gude, S Guenter and S Sesnic 1999 Nucl. Fusion 39127

[11] RJ Buttery et al 2004 Nucl. Fusion 44678

[12] O Sauter et al 2002 Phys Rev Lett. 88105001

[13] S Coda et al 2007 in Proceedings of the 34th EPS Conference on Controlled Fusion and Plasma Physics, Warsaw P5.130

[14] CC Hegna, JD Callen and RJ La Haye 1999 Phys. Plasmas 6130

[15] MFF Nave et al 2003 Nucl. Fusion 43179

[16] H Reimerdes et al 2002 Phys. Rev. Lett. 88105005

[17] P Maget et al 2005 Plasma Phys Control Fusion 47357

[18] HR Koslowski et al 2000 Nucl. Fusion 40821

[19] RJ Buttery et al 2003 Nucl. Fusion 4369

[20] O Sauter et al 1997 Phys. Plasmas 41654

[21] PA Belo et al 2001 in Proceedings of the 28th EPS Conference on Controlled Fusion and Plasma Physics, Portugal P5.004

[22] IT Chapman 2010 sub Plasma Phys. Control. Fusion "Controlling sawtooth oscillations in tokamak plasmas" 
[23] Porcelli F, Boucher D and Rosenbluth M 1996 Plasma Phys. Control. Fusion 382163

[24] DJ Campbell et al 1988 Phys. Rev. Lett. 602148

[25] ITER Physics Basis 1999 Nucl. Fusion 392137

[26] SC Jardin, MG Bell and N Pomphrey 1993 Nucl. Fusion 33371

[27] G Bateman et al 1998 Phys. Plasmas 52355

[28] R Waltz et al 1997 Phys Plasmas 42482

[29] T Onjun and Y Pianroj 2009 Nucl. Fusion 49075003

[30] RV Budny et al 2008 Nucl. Fusion 48075005

[31] YR Martin et al 2008 Journal of Physics: Conference Series 123012033

[32] O Sauter, C Angioni and YR Lin-Liu 1999 Phys Plasmas 62834

[33] RJ La Haye et al 2000 Phys. Plasmas 73349

[34] JP Graves et al 2009 Phys. Rev. Lett. 102065005

[35] L-G Eriksson et al 2006 Nucl. Fusion 46 S951

[36] EJ Doyle et al Progress in the ITER Physics Basis Chapter 2: Plasma confinement and transport 2007 Nucl. Fusion 47 S18

[37] Y Gribov, ITER Organisation 2009 Private Communication

[38] C Zucca et al 2008 Theory of Fusion Plasmas, Joint Varenna-Lausanne Theory Conference 1069361

[39] C Zucca 2009 PhD Thesis No 4360, EPFL, Lausanne "Modeling and control of the current density profile in tokamaks and its relation to electron transport"

[40] P Maget et al 2005 Plasma Phys. Control. Fusion 47357

[41] RGD Steel and JH Torrie, 1960 "Principles and Procedures of Statistics" (New York: McGraw-Hill) p187

[42] IT Chapman et al 2007 Plasma Phys. Control. Fusion 49 B385 